\title{
Editorial - Special Issue on Collaboration Support Systems (CSS)
}

\author{
H.S. Ko, S. Y. Nof
}

Recent and emerging advances in computer and information science and technology have realized a powerful computing and communication environment. It enables effective interactions and collaboration among groups of people and systems (and systems-of-systems) beyond traditional restrictions of time and space. The evolution in hardware (e.g., pervasive computing devices, wireless sensor networks, nano-electronics) and software (e.g., multi-agent systems, workflow and information integration, interaction models and protocols) technology, and their flexible teaming have further enabled diverse forms of collaboration approaches. It has been observed during the last few decades that numerous collaboration methodologies, tools and applications in various domains have emerged to provide better quality services, helping to solve domain-specific, highly complex problems. The development of collaboration tools and methodologies has increased the domain knowledge that can be discovered and shared by individuals, and the level and intensity of interactions and collaboration that can dramatically decrease problem complexity and increase solution quality. At the same time, inefficient interactions, task and information overloads, and ineffective collaboration are prevalent.

In spite of the considerable progress in collaboration tools and methods, a sound foundation of collaboration science is yet to be established; lacking such foundation is a major obstacle in leveling up sophistication and anticipated benefits of collaboration methods. Recently, however, Collaboration Control Theory (CCT) models and techniques have been proposed as the foundation of designing Collaboration Support Systems (CSS), and new features of CSS are being investigated in various areas based on CCT. In order to provide readers with a significant opportunity of investigating novel and forthcoming problems in collaboration research, the guest editors have invited authors from various disciplines and focused on collaborative design and modeling features in the respective areas. After careful and rigorous review and revision processes, eight articles have been selected for their special quality and relevance to CSS. The selected articles are organized along two main topics in CSS: 1) CSS Models and Theories; and 2) CSS Methods and Applications.

In the area of CSS Models and Theories:

- "Swarming models for facilitating collaborative decisions" - Zamfirescu and Filip introduce the use of swarming models (stigmergic mechanisms) to build collaborative support systems for complex cognitive tasks, exemplifying them through an experiment for group decision processes (GDP) in e-meetings.

- "Design of Protocols for Task Administration in Collaborative Production Systems" - Ko and Nof investigate the design of task administration protocols for collaboration support in production system, where those protocols, as control mechanisms, can manage complicated events in the collaborative task workflow environment and overcome limitations of coordination protocols.

- "Mining authoritativeness of collaborative innovation partners" - Engler and Kusiak, present a novel approach to automatically determine the authoritativeness of entities for collaboration and demonstrate the use of mining schema for identifying collaboration partners over the Internet.

- "Reference architecture for collaborative design" - Huang, Yang, Chen, and Nof present reference architecture for collaborative design (CD) as a framework for analyzing and supporting CD, then describe and illustrate dimensions forming the architecture as a cube of design aspect, design stage, and collaboration scope.

The next group of articles focuses on CSS Methods and Applications: 
- "Coordinating aerial robots and unattended ground sensors for intelligent surveillance systems" - de Freitas, Heimfarth, Allgayer, Wagner, Larsson, Pereira, and Ferreira present a system solution to enable interoperability and coordination support for heterogeneous sensor networks composed of low-end ground sensor nodes and mobile sensors carried by autonomous aerial robots.

- "Introducing collaborative practices in small medium enterprises" - Antonelli and Chiabert propose a methodology to evaluate the possibility of using PLM as a framework which exploits collaboration links within an enterprise, based on an exhaustive analysis of the PLM impact on different aspects of the enterprise.

- "A software system development life cycle model for improved stakeholders' communication and collaboration" - Cohen, Dori, and De Haan describe a collaborative software system development life cycle model using Object Process Methodology (OPM), which includes various stakeholders and variables, and considers multiple aspects in collaboratively developing off-theshelf software.

- "Gaze, posture and gesture recognition to minimize focus shifts for intelligent operating rooms in a collaborative support system" - Wachs describes the design of an intelligent, collaborative system framework, which involves the integration of machine vision, voice recognition and computer graphics-projection techniques to improve operation rooms for surgery by highly intuitive, natural and multimodal interactions.

These articles are presented based on related scientific investigations around the world and reflect well on the broad and challenging area of CSS. The guest editors wish to thank all the contributing authors, the referees, and the editorial office colleagues who have all endeavored to bring this special issue on CSS to you.

H.S. Ko and S.Y. Nof West Lafayette, Indiana, USA Special Issue Guest Editors 